\title{
Quality Management System in Radiotherapy Needs and Requirements
}

\author{
K. El-Ghamrawi, A. Seleem, M. El-Haddad and E. Mahmoud \\ Clinical Oncology Department Faculty of Medicine, Cairo University
}

\begin{abstract}
Radiotherapy is one of the main treatment modalities in cancer management. Over $50 \%$ of cancer patients will receive radiotherapy (RT) at least once during their treatment. It plays an important role in the treatment of $40 \%$ of those patients who are cured ${ }^{1}$.

Radiation treatment involves many health professionals, sophisticated machines and complicated techniques, working through an integrated process to plan and deliver radiation to cancer patients. Radiotherapy aims at highest tumor control probability (TCP) with lowest morbidity rates. For these goals the establishment and use of comprehensive quality management system (QMS) is highly required to meet these objectives. These needs became more important and strongly required since the introduction of high precision radiation techniques as stereotaxy, 3D conformal, intensity modulated and tomotherapy.

Historically, actions summarized under the term "quality assurance" (QA) has long been carried out in many RT services since ${ }^{2}$ or even ealier. They were restricted to physical and technical aspects of equipments, dosimetry and treatment delivery. Nowadays the concept is much broader and encompass a comprehensive approach to all activities in RT department, thus it has clinical, physical and administrative components ${ }^{3}$.
\end{abstract}

Key words: Radiotherapy, Quality Assurance, Comprehensive Quality Management. Corresponding author: K. El-Ghamrawi

E-mail: K.El-Ghamrawi@yahoo.com

\section{INTRODUCTION}

In this context, several terms are better defined to minimize confusion and to facilitate interdepartmental comparisons.

"Quality standards" (QS): Are the set of accepted criteria against which the quality of activity in question can be assessed. Various national and international organizations have issued recommendations for standards in RT. 4,5,6,7,8. Every radiotherapy department should define its own QS in its quality manual.

"Quality controls" (QC): Referes to the regulatory process through which the actual quality performance is measured, compared with the existing QS and the actions necessary to keep or to regain conformance with the stardards. QC is one port of overall QA and includes many subactivaties as document control, procedures to ensure that the quality system is followed, reporting all non-conforming parts and taking corrective actions ${ }^{5}$.

"Quality Assurance" (QA): In health care system means all activities and programmes intended to assure or improve the quality of medical care ${ }^{9}$.

"QA in radiotherapy" is conserved with all aspects of radiotherapy process and includes all groups of staffing in cooperative approach, since quality activities are interdependent starting from the moment patient arrives at RT department till he completes treatment and through his follow-up period ${ }^{10}$.

"Comprehensive Quality Management System" (CQMS): It is a comprehensive quality program built on quality manual (QM).

"Quality Manual" (QM): Is the official document that declares the specific quality practices, resources available, and activities related to patient care services it provides. This document should cover the quality of all aspects of patient care in RT department including personnel qualifications, equipment performance, fabrication process, utilization process and documentation of all aspects of radiation delivery services. In this QM document, the QA checks, frequency of checks, action criteria, records of checks and personnel responsible for performing these checks must be clearly defined. The QA program must be sufficiently comprehensive so that the commulative effects of uncertainties associated with complete RT process can be determined. This allows tolerance levels and thereafter action criteria can be set for parameters that are being checked ${ }^{10}$. However it has to 
be mentioned that the formulation of QA guidance lags far behind the rapid penetration of new technologies and machines leaving the practioners and patients at greater risk of catastrophic delivery errors. Therefore it may be a wise policy for RT departments specifically in less-developed countries to slow down in purchasing first generation machines or performing new techniques before making sure of their safety and availability of adequate QA guidance ${ }^{11}$.

The ultimate goal of any CQMS in RT is to improve quality of care and treatment effectiveness. This goal is usually achieved by the following objectives:

Ensuring proper adaptation of treatment strategy (clinical aspects of QA which includes among other items treatment protocols).

- Maintaing the required accuracy in RT.

- Minimizing the risk of error and accidents through proper safety standards.

- Continuing quality improvement and increase efficiency.

- Recognition of CQMS as a management tool.

- Cultural change of personnel.

- Reduction of chance of litigation.

- Guaranteeing the validity of clinical trials ${ }^{12}$.

In order to achieve the above mentioned objectives, the following series of tasks has to be accomplished:

1. Formulation of department's policy. The head of the department with its senior staff have to define vision, objectives and basic goals of the department in a formal document called the "Policy Manual".

2. Creation of a QM project team and QM committee. The team will be responsible for performing QA related tasks and should reflect the multidisciplinary RT team. The committee is appointed by the head of department with full authority to manage QA, approve QA policies and procedures and the assignment of QA responsibilities in the department.

3. Structure of quality system preferably in 3 hierarchial levels which should be outlined in the QM:

- Level 1: Reflects definition of its objectives, strategies developed to meet these objectives and responsibilities of supervision of all functions (policy of the department).
- $\quad$ Level 2: Refers to all procedures for which a formal organization is required. This includes the scope of procedure, responsibilities of these involved and the outline of the practical action.

- Level 3: covers the work instruction ${ }^{13}$.

4. Description of the department structure: this item covers an organogram and communication, department infrastructure and process contro ${ }^{15}$.

5. Development of QM (see before).

6. Implementation which covers training and validation phase.

7. Quality audit (internal and external) and continuous monitoring.

8. Quality system for RT equipment (Mean and Material).

It can not be emphasized more that such CQMS should be reviewed at regular intervals to be modified and or updated according to the regular reports issued by the quality committee and in view of recent data reported in this context.

It is recommended that the national RT organization should take the task of introducing the concept CQMS to all RT departments across the country and to give technical assistance in its implementation.

\section{REFERENCES}

1. World Health Organisation (WHO). Radiotherapy risk profiles technical manual.2008 World Health Organisation (WHO), Geneva.

2. Bernier J, Horiot JC, Poortmans P. Quality assurance in radiotherapy: From radiation physics to patient and trial-oriented control procedures. Eur.J.Cancer 2002 Mar;38(Suppl 4):S155-8.

3. Hartmann G. Quality management in radiotherapy. In: Schlegel WC, Bortfeld T, Grosu AL, editors. Berlin: Springer; 2006. pp. 425-47.

4. Kutcher GJ, Coia L, Gillin M, Hanson WF, Leibel S, Morton RJ, et al. Comprehensive QA for radiation oncology: Report of AAPM Radiation Therapy Committee Task Group 40. Med.Phys. 1994 Apr;21(4):581-618.

5. Makenzie A. NATO science for peace and security series B: Physics and biophysics.2009 Part 11. 71-79. Springer.

6. IAEA. Comprehensive audits of radiotherapy practices: A tool for quality improvement, Quality Assurance Team for Radiation Oncology. Vienna: IAEA; 2007.

7. World Health Organisation (WHO). International classification for patient safety. Geneva: World Health Organisation (WHO); 2008.

8. Thwaites DI, Mijnheer BJ, Mills JA. Quality assurance of external beam. Radiation oncology physics: A handbook 
for teachers and students Vienna: International Atomic Energy Agency; 2005. pp. 407-50.

9. Mondofacto. Quality assurance, health care definition. 2009; Available: http://www.mondofacto.com/facts/ dictionary.

10. Saw C, Ferenci M, Wanger HJ. Technical aspects of quality assurance in radiation oncology. Biomed.Imaging Interv.J. 2008 Jul;4(3):e48.

11. Ishikura S. Quality assurance of radiotherapy in cancer treatment: Toward improvement of patient safety and quality of care. Japan.J.Clin.Oncol. 2008;38(11):723-9.

12. Hartmann GH. Accuracy strategies in radiotherapy. NATO Science for Peace and Security Series B: Physics and Biophysics 2009:11-27.

13. Leer JW, Mckenzie A and Scalliet P. Practical guide lines for implementation of quality system in radiotherapy. 1998 ESTRO booklet No. 4. Brussels, Belgium. 
Kasr-El-Aini Journal Of Clinical Oncology And Nuclear Medicine

Vol. 7 | No. 1-2 2011

Quality Management System in Radiotherapy Needs and Requirements 
